Rapid Reviews COVID-19•

\title{
Review 1: "Rapid Detection of SARS-CoV-2 Antibodies Using Electrochemical Impedance-Based Detector"
}

Rebecca DuBois ${ }^{1}$, John Dzimianski ${ }^{1}$

${ }^{1}$ University of California Santa Cruz Jack Baskin School of Engineering Biomolecular Engineering UNITED STATES

Published on: Oct 01, 2020

License: Creative Commons Attribution 4.0 International License (CC-BY 4.0). 


\section{$\underline{\text { RR:C19 Evidence Scale rating by reviewer: }}$}

- Potentially informative. The main claims made are not strongly justified by the methods and data, but may yield some insight. The results and conclusions of the study may resemble those from the hypothetical ideal study, but there is substantial room for doubt. Decision-makers should consider this evidence only with a thorough understanding of its weaknesses, alongside other evidence and theory. Decisionmakers should not consider this actionable, unless the weaknesses are clearly understood and there is other theory and evidence to further support it.

*****************************************

\section{Review:}

\section{Summary}

Rashed et al. present exploratory research into the use of electrochemical impedance for the detection of SARS-CoV-2 antibodies in serum. Using the antibody CR3022, which has known cross-reactivity with the SARS-CoV-2 spike RBD, the authors validate the approach in principle. Following this, they tested six clinical serum samples using their method and showed a general correlation with the results obtained by ELISA. While this is interesting research with potentially exciting applications, the conclusions should be viewed as tentative due to intrinsic error in the technique and limitations in the study design and reported data.

\section{Specific Comments}

The major concerns with the manuscript are the (1) intrinsic error in the measurements, (2) the lack of some experimental controls, and (3) only partial reporting of data. The error bars in Figure 2B show substantial overlap between the different CR3022 concentrations, suggesting that single-point sample testing (as is typical for initial screening) may yield inaccurate and/or misleading results. The lack of negative control in Figure 2 also makes it unclear what the potential lower limit of detection may be-thus the potential for false positives and negatives in this methodology. Similar issues are present in Figure 3-the data for clinical samples also have large error margins. Furthermore, while a negative sample is included in 3A, it is unclear whether any of those shown in 3B could be negative. It is puzzling as to why, with only six samples, the authors did not present data for all of them. While the small sample size itself presents caveats (but could be improved with more samples, 
especially with the commercial availability of convalescent plasma), the lack of important controls, statistical analyses, and incomplete reporting of the data is a concerning issue that creates a great deal of ambiguity.

Minor concerns:

- Referring to CR3022 as a "SARS-CoV-2 monoclonal antibody" is imprecise, as this antibody came from a patient from the 2002-2003 SARS outbreak. While crossreactive for the SARS-CoV-2 spike, studies during the first months of the COVID-19 pandemic suggested that it does not neutralize SARS-CoV-2 (e.g. Yuan et al. 2020, Science).

- It appears that a potentially important reference may have been overlooked during proofreading (“...protein/antibody binding events will influence the EDL resistance and capacitance at low frequencies (?) and...").

- Figure 1 panel labels don't match up to the legend.

- The statement on page 3 regarding antibody quantitation is not supported by the data: "impedance spectroscopy can be used to quantify antibody levels in serological samples".

- Figure 3B: it is not clear what the samples are (seronegative or seropositive). Also, ELISA data is missing error bars. 